\title{
Perivascular adipocytes and signaling through toll-like receptors: role in the pathophysiology of atherosclerosis
}

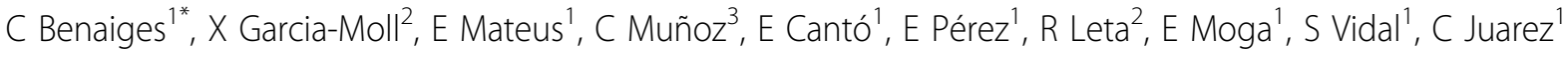 \\ From 5th European Workshop on Immune-Mediated Inflammatory Diseases \\ Sitges-Barcelona, Spain. 1-3 December 2010
}

\section{Introduction}

Perivascular adipose tissue has emerged as a critical regulator of vascular function implicated in the pathophysiology of atherosclerosis. Virtually all arteries are surrounded by a significant amount of perivascular adipose tissue. This tissue is a very active endocrine and paracrine source of inflammatory cytokines and adipokines. Moreover, adipocytes express toll-like receptors (TLRs) to be able to respond to lipids and other self and nonself molecules activating proinflammatory pathways. We analyze the TLR/JAK-STAT/SOCS transduction pathways in adipose tissue from patients with atherosclerosis to unravel the mechanisms implicated in the pathophysiology of atherosclerosis.

\section{Materials and methods}

Perivascular and subcutaneous adipose tissue was obtained from atherosclerosis and control patients. Adipose tissue was cultured in the presence of TLR ligands to analyze the activation of TLR/JAK-STAT pathways. Expression and activation levels of STATs were measured by western blot and immunohistochemistry analysis using specific anti STATs antibodies. The secretion of adipokines (Adiponectin, Resistin and Leptin) and cytokines (IL-6, IL-10 and TNF) in supernatants were quantified by ELISA.

\section{Results}

Western blot and immunohistochemistry analysis of STAT proteins upon stimulation of adipose tissue by TLR ligands showed an increased expression of STAT-1, STAT-3, Phospo-STAT1, Phospo-STAT3 and PhospoSTAT5.
Cytokine secretion increased in those supernatants that were stimulated with TLR ligands, especially IL-6 secretion. Adipokine secretion did not show significant variations after stimulation.

\section{Conclusions}

We identified alterations in the TLR/JAK-STAT signaling pathways in adipose tissue that would help to explain the deleterious effects of lipids and other TLR ligands in the pathophysiology of atherosclerosis. The results will be very useful to understand the role played by adipose tissue in atherosclerosis and to design therapeutic approaches to control this inflammatory process.

\section{Author details}

${ }^{1}$ Department of Immunology, Hospital de la Santa Creu i Sant Pau, Universitat Autonoma de Barcelona, Barcelona, Spain. 'Department of Cardiology, Hospital de la Santa Creu i Sant Pau, Universitat Autonoma de Barcelona, Barcelona, Spain. ${ }^{3}$ Cardiac Surgery, Hospital de la Santa Creu i Sant Pau, Universitat Autonoma de Barcelona, Barcelona, Spain.

Published: 25 November 2010

doi:10.1186/1479-5876-8-S1-P23

Cite this article as: Benaiges et al:: Perivascular adipocytes and signaling through toll-like receptors: role in the pathophysiology of atherosclerosis. Journal of Translational Medicine 2010 8(Suppl 1):P23. 